\title{
LOWER LEG PERFORATORS IN HUMAN HEALTH AND DISEASE
}

\author{
Marian Simka \\ Department of Nursing, College of Applied Sciences, Ruda Śląska, Poland
}

\author{
EDITORIAL COMMENTARY \\ Phlebological Review 2014; 22, 2: 49 \\ DOl: $10.5114 /$ pr.2014.48904
}

In this issue's review paper Čestmir Reček meticulously describes the activity of lower leg perforators in physiological settings and in patients with chronic venous disease [1]. Despite the importance of the role played by these veins for proper functioning of the venous system of the low extremities, their pathophysiology is still poorly understood. This leads to improper management of patients with chronic venous insufficiency.

Unfortunately, our understanding of this problem is unlikely to be substantially improved in the near future. At the moment, most of the research in phlebology is based on Doppler sonographic examinations. Nevertheless, even if the accuracy of this diagnostic tool is improving, this imaging technique is not very useful in resolving uncertainties related to the pathophysiology of venous outflow from the lower extremities. Direct pressure measurements in particular veins combined with anatomical assessment of these blood vessels and evaluation of the flow characteristics would be more reliable. However, considering the invasive nature of such studies, for ethical reasons they are not likely to be performed in the foreseeable future. Thus, we should rely on the results of research performed several decades ago [2-9]. However, becuase these studies usually examined a single physical parameter (for example: pressure), many questions remain unanswered.

It should be emphasised that - contrary to common thinking - blood flow is not driven by the difference in pressure between two points, but by the difference of total energy between these two points. This transmission of energy can be described using Bernoulli's principle, which states that for an ideal fluid the function describing the total energy of such a fluid is constant along a streamline [10]. This total energy consists of the sum of the pressure energy (associated with the pressure), the kinetic energy (associated with flow velocity), and the potential energy (associated with gravitation).

Going back to the above-mentioned research on pressure changes in the leg veins, it is unclear how the other components of Bernoulli's equation (especially the kinetic energy, i.e. flow velocity) were changing during these experiments. In addition, according to Bernoulli's equation, the result of the pressure measurement in the blood vessel will be different depending upon how the pressure is measured. For example, if a catheter has an end-port sensor that is facing the flowing stream of the blood, it will measure a pressure that is significantly higher than the pressure measured by a side-port sensor on the same catheter, while the measured pressure will be even lower if such a sensor is positioned against the flow direction. This difference between the types of pressure measurements can range from a few $\mathrm{mmHg}$ to more than $20 \mathrm{mmHg}$, depending on the velocity of the flowing blood. It is unclear how the pressure was measured in the above-cited historical experiments.

It should also be remembered that the Bernoulli principle is only applicable to non-viscous fluids. Moreover, for Bernoulli's principle it is assumed that no work is being done on the fluid (which is not the case of a living organism). Therefore, in the case of blood flow Bernoulli's equation is only an approximation of the real world, and to describe the flow more accurately much more complicated Navier-Stokes equations should be used [10].

Thus, for the time being it is not known how to bridge the gap in understanding the behaviour of the lower leg perforators. Nevertheless, contemporary phlebologists, equipped exclusively with Doppler sonography and ignoring physical laws governing the flow mechanics, are unlikely to comprehend fully the phenomena occurring during blood flow in the veins of the lower extremities.

\section{References}

1. Reček Č. The haemodynamic role of calf perforators. Phlebol Rev 2014; 22: 45-48.

2. Arnoldi C.C. Venous pressure in the legs of healthy human subjects at rest and during muscular exercise in nearly erect position. Acta Chir Scand 1965; 130: 570-583.

3. Bjordal R.J. Simultaneous pressure and flow recording in varicose veins of the lower extremity. Acta Chir Scand 1970; 136: 309-317.

4. Bjordal R.J. Circulation patterns in incompetent perforating veins in the calf and the saphenous system in primary varicose veins. Acta Chir Scand 1972; 138: 251-261.

5. Burnard K.G, O’Donnell J.T, Thomas M.L. The relative importance of incompetent communicating veins in the production of varicose veins and venous ulcers. Surgery 1977; 82: 9-13.

6. Höjensgard I.C., Stürup H. Static and dynamic pressures in superficial and deep veins of the lower extremity in man. Acta Physiol Scand 1952; 27: 49-67.

7. Reček Č., Koudelka V. Effect circulatoire du reflux saphénien dans la varicose essentielle. Phlebologie 1979; 32: 407-414.

8. Reček Č. A critical appraisal of the role of ankle perforators for the genesis of venous ulcers in the lower leg. J Cardiovasc Surg (Torino) 1971; 12: 45-49.

9. Sarin S., Scurr J.H., Coleridge Smith P.D. Medial calf perforators in venous disease: the significance of outward flow. J Vasc Surg 1977; 16: 40-46.

10. Kambe T. Elementary fluid mechanics. World Scientific Publishing Co. Pte. Ltd., Singapore 2007. 